\title{
Hydration of Ions in Confined Spaces and Ion Recognition Selectivity
}

\author{
Tetsuo OKADA, ${ }^{\dagger}$ Makoto HARAdA, and Takumi OHKI \\ Department of Chemistry, Tokyo Institute of Technology, Meguro, Tokyo 152-8551, Japan
}

\begin{abstract}
The hydration of ions in confined spaces, such as the interior of ion-exchange resins, micelles, and surface monolayers, is discussed on the basis of results obtained with X-ray absorption fine structure studies, electrophoresis, and ion-transfer voltammetry. The general trends are that anions are partly dehydrated therein, whereas cations are likely to keep their first hydration shells. For bromide ions, the hydration numbers under various circumstances have been determined. The extents of dehydration depend not only on the structure of the cationic sites electrostatically attracting bromide ions but also on whether the cationic sites are exposed to a solution or are effectively shielded from it. These findings will be useful for designing the systems for ionic recognition and separation.
\end{abstract}

(Received August 27, 2008; Accepted October 2, 2008; Published February 10, 2009)

\begin{tabular}{|c|c|c|c|}
\hline 1 Introduction & 167 & 3 Hydration of Ions in Self-assembled Systems & 171 \\
\hline 2 Hydration of Ions in Ion-Exchange Resins & 168 & 3.1 Binding of ions to micelles and their & \\
\hline $\begin{array}{l}2 \cdot 1 \text { General aspects of separation selectivity of } \\
\text { ion-exchange }\end{array}$ & & $\begin{array}{l}\text { hydration structures in micelles } \\
\text { 3.2 Hydration of ions in surface monolayers }\end{array}$ & \\
\hline 2.2 Local structures of counterions in dried resins & & 4 Ion Recognition Selectivity & 174 \\
\hline 2.3 Hydration structures of counterions in ion- & & 5 Conclusion & 174 \\
\hline exchange resins & & 6 References & 174 \\
\hline
\end{tabular}

\section{Introduction}

Modern technologies have allowed us to access the molecular processes taking place in confined spaces that are formed using various classes of materials, such as porous solids or gels, layered materials, and self-assembled molecular aggregates. It is known that materials confined in nanospaces show structures and/or dynamics different from those exhibited in bulk. ${ }^{1-11}$ Such unusual properties, which become more apparent as the size of the space decreases, partly come from the effects of the walls surrounding the spaces. ${ }^{2,6}$ The interaction of compounds with the wall becomes more dominant with decreasing size of the space. In addition, molecular aggregates and clusters cannot retain their stable sizes in such narrow spaces and show unusual physicochemical features as a result. Various compounds are accommodated in confined spaces, and their molecular structures and dynamics therein have been studied by X-ray and neutron scattering, ${ }^{3-5}$ spectroscopy, ${ }^{6,7}$ and computer simulations. ${ }^{9,10}$

An interface, which can be considered as a space twodimensionally confined between two phases, has received increasing attention in analytical sciences. ${ }^{10,12,13}$ A special issue of Analytical Sciences in 2004 was devoted to the compilation of papers treating liquid-liquid interfaces. ${ }^{13}$ An interface is usually difficult to approach by usual methods, because it

$\dagger$ To whom correspondence should be addressed.

E-mail: tokada@chem.titech.ac.jp comprises an extremely small sector in the entire condensed phase system, and, in turn, the amounts of molecules concerned are also very small. Thus, the signals in usual measurements mostly come from the bulk phase rather than from the interface. High interfacial selectivity is thus undoubtedly required to probe the phenomena of our interest; interfacial measurements are obviously a challenge for analytical scientists. On the other hand, we have consciously or often unconsciously utilized interfaces in designing analytical methods such as separation and recognition of various molecules, and, from this perspective, have been strongly involved in interfacial sciences for a long time. Analytical scientists are expected to continue to contribute to the further understanding of chemistry in interfacial and nanospace chemistry by devising new methodologies as well as by developing useful applications.

In the separation and recognition of ions, the design of molecules with specific selectivity is usually regarded as essential. Although this approach has proven useful for ions forming inner-sphere complexes, it is doubtful whether it is applicable to the outer-sphere interactions of a number of ions such as alkali, alkaline earth cations, and anions. For these ions, it is more important that their local structures are explicitly evaluated at the place where recognition occurs, mostly interfaces and nanospaces. The essential involvements of solvation have long been recognized in this area. ${ }^{142}$ Therefore, the solvation or crystalline sizes of ions have often been mentioned in the discussion of ion recognition selectivity. The recent advancements of molecular architectures have allowed 
the preparation of highly controlled nanospaces, into which ions would be partitioned in solvated or desolvated forms. However, since direct experimental studies of ions are, in general, difficult when small spaces should be probed, the ion selectivity is mostly discussed on the basis of molecular simulations. ${ }^{9,24-27}$

We have been involved in the elucidation of the molecular origins of selectivity in separation of ions, and have employed chromatography, electrophoresis, X-ray absorption fine structure (XAFS), and ion-transfer voltammetry to evaluate the structures of ions and the thermodynamics concerned with separation. ${ }^{14}$ Separation provides highly reliable and reproducible results originating from multistep interfacial interactions; therefore, efficient designs of the system suitable for e.g. spectroscopic measurements are possible on the basis of information obtained from separation work. According to this idea, we have employed XAFS measurements, the systems for which were designed after chromatographic separation, electrophoresis, and ion-transfer voltammetry. Although XAFS is a powerful tool to probe the local structures of ions, we measure the average structures of ions present in the path of the incident X-ray. Measurements and/or samples should be reasonably modified to employ this method as a nanospace-selective approach. In this review, we focus our attention on the solvation of ions in confined spaces formed by materials used for separation and recognition of ions, and discuss this topic in relation to ion selectivity.

\section{Hydration of Ions in Ion-Exchange Resins}

2.1 General aspects of separation selectivity of ion-exchange

An ion-exchange resin is usually composed of cross-linked polymers with charged groups immobilized along the macromolecular skeleton. Although the interior of an ionexchange resin that is well swollen in a solvent is sometimes considered as a concentrated polymer solution, we here consider that both ionic groups and counterions are confined in small spaces surrounded by polymer chains. Such models have been well established for a cation-exchanger membrane, Nafion. ${ }^{28-30}$ Ion-exchange selectivity is mainly determined by two factors: i.e. the charge of an ion and its solvation. Since the interaction between ions and ion-exchange groups on resins is electrostatic, the charge on an ion is in general accepted as a dominant factor governing separation selectivity of ion-exchange. However, this intuitively acceptable mechanism is often given too much emphasis on the discussion of ion-exchange selectivity; the second effect plays important roles in some instances.

Identically charged ions can be separated with ion-exchange, which provides selectivity large enough to allow systematic separation of e.g. a number of monovalent anions. In some cases, monovalent ions are more strongly captured than divalent counterparts by ion-exchange resins. ${ }^{31}$ These facts cannot be interpreted solely by electrostatic interactions, suggesting the involvements of other factors. The hydration of ions has been considered as a factor affecting the separation selectivity in ion exchange. ${ }^{14-19}$ The Donnan theory for ion-exchange explains the ion-exchange selectivity between identically charged ions on the basis of their molar volumes. ${ }^{32}$ Also, the hydration of counterions in ion-exchange resins was studied in details on the bases of the water adsorption isotherms on the resins. ${ }^{16-19}$ Since the thermodynamic discussions have obvious limitations, structural information has been a keen demand for further discussions of this very common phenomenon. The first question will be whether counterions are directly bound by the ion-exchange active groups or not. The second one is to what extent the counterions are hydrated. The hydration number of

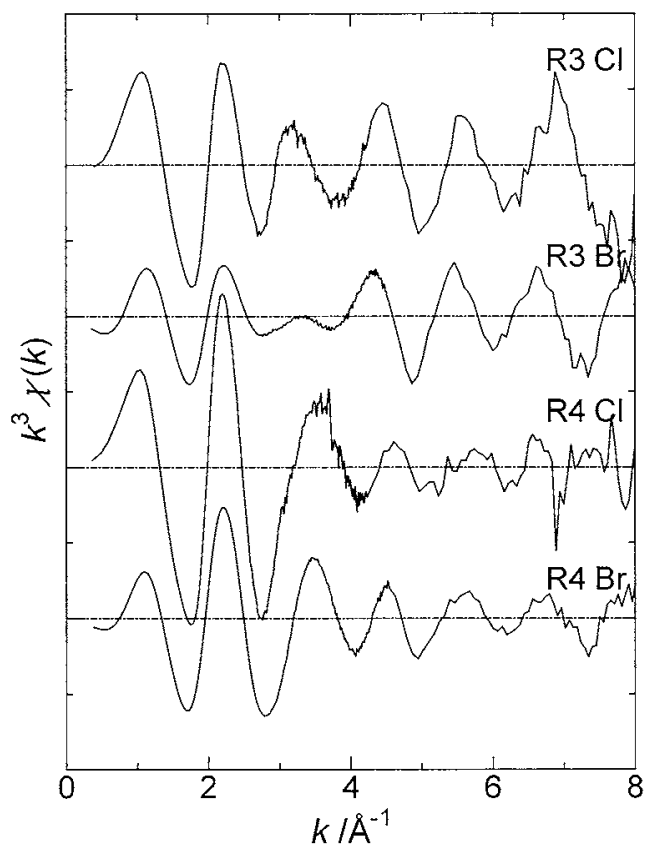

Fig. 1 XAFS $\chi$ spectra for dried resins. Reproduced with permission from Ref. 33.

counterions should be smaller than that in bulk water if the ions are bound by the ion-exchange groups, whereas the first coordination shell should be preserved when counterions are dissociated from the active sites.

\subsection{Local structures of counterions in dried resins}

Before the discussion of hydrated counterions in an ionexchange resin, it will be helpful to see their local structures in the dried resin, in which counterions should be directly bound by ion-exchange sites. XAFS spectra were measured for $\mathrm{Cl}^{-}$and $\mathrm{Br}^{-}$in two types of anion-exchange resins with trimethylammonium (R4) or dimethylammonium (R3) groups as ion-exchange sites ${ }^{33}$ as well as for $\mathrm{K}^{+}, \mathrm{Rb}^{+}$, and $\mathrm{Sr}^{2+}$ in a cationexchange resin with sulfonate groups. ${ }^{34}$ Figure 1 shows the XAFS $\chi k^{3}$ spectra for $\mathrm{Cl}^{-}$and $\mathrm{Br}^{-}$in the dried $\mathrm{R} 4$ and $\mathrm{R} 3$ resins. The oscillation intensity for R4 simply decreases with increasing $k$, suggesting that scattering groups are present only in the first coordination shell of the counteranions. In contrast, the oscillation intensity for $\mathrm{R} 3$ first decreases with increasing $k$, but again increases when $k$ exceeds $4 \AA^{-1}$. The increase in the oscillation intensity at $k>4 \AA^{-1}$ is ascribed to the formation of a well-defined second coordination shell. The XAFS parameters determined by curve-fitting are also summarized in Table 1. The number of scattering atoms in the $\mathrm{R} 4$ resin was estimated to be $c a$. 4 for both $\mathrm{Cl}^{-}$and $\mathrm{Br}^{-}$, indicating that the anions sit on a tripod consisting of three methyl groups (the upper figure in Fig. 2). A molecular model indicated that the distance between the halide anion and the methyl carbon atoms is slightly shorter than that between the anion and the ammonium nitrogen ( $c a$. $0.2 \AA$ ). However, the counterions move around inside of the tripod, and, in addition, carbon and nitrogen atoms have similar backscattering abilities; these similarities make difficult to distinguish them. The number of the scattering atoms ( $\mathrm{ca}$. four) is thus understandable for the structure illustrated in Fig. 2. In contrast, the results for the R3 resins are slightly complicated. The oscillation intensity for the $\mathrm{R} 3$ resins first decreases with increasing $k$, but again increases when $k>4 \AA^{-1}$, suggesting a well-defined second coordination shell. If the halide anions 
Table 1 Structural parameters for $\mathrm{Cl}^{-}$and $\mathrm{Br}^{-}$in the dried $\mathrm{R} 3$ and $\mathrm{R} 4$ resins ${ }^{33}$ and for $\mathrm{K}^{+}, \mathrm{Rb}^{+}$, and $\mathrm{Sr}^{2+}$ in dried cation-exchange resins $^{34}$

\begin{tabular}{ccccc}
\hline Resin & Scattering atom & $N^{\mathrm{a}}$ & $r / \AA^{\mathrm{b}}$ & $\sigma / \AA^{\mathrm{c}}$ \\
\hline $\mathrm{R} 3 \mathrm{Cl}$ & $\mathrm{N}$ & 1.08 & 3.02 & 0.13 \\
& $\mathrm{C}$ & 3.12 & 3.58 & 0.19 \\
$\mathrm{R} 3 \mathrm{Br}$ & $\mathrm{C}($ or N) & 2.03 & 3.27 & 0.11 \\
& $\mathrm{C}$ & 2.24 & 3.41 & 0.14 \\
$\mathrm{R} 4 \mathrm{Cl}$ & $\mathrm{C}$ (or N) & 4.52 & 3.27 & 0.25 \\
$\mathrm{R} 4 \mathrm{Br}$ & $\mathrm{C}$ (or N) & 4.27 & 3.53 & 0.20 \\
$-\mathrm{SO}_{3} \mathrm{~K}$ & $\mathrm{O}$ & 2.75 & 3.13 & 0.16 \\
$-\mathrm{SO}_{3} \mathrm{Rb}$ & $\mathrm{O}$ & 2.73 & 3.67 & 0.17 \\
$-\mathrm{SO}_{3} \mathrm{Sr}$ & $\mathrm{O}$ & 2.47 & 3.67 & 0.08 \\
\hline
\end{tabular}

a. The number of scattering atoms.

b. The distance between the absorbing and scattering atoms. c. Debye-Waller coefficient.

Fitting ranges of $k$; K $K$-edge $\left(k=2.0-7.0 \AA^{-1}\right), \operatorname{Rb} K$-edge $(k=1.8$ $\left.5.0 \AA^{-1}\right)$, Sr $K$-edge $\left(k=2.0-10.0 \AA^{-1}\right), \mathrm{Cl} K$-edge $\left(k=1.5-5.5 \AA^{-1}\right)$, and $\mathrm{Br}-K$ edge $\left(k=1.5-6.5 \AA^{-1}\right)$.
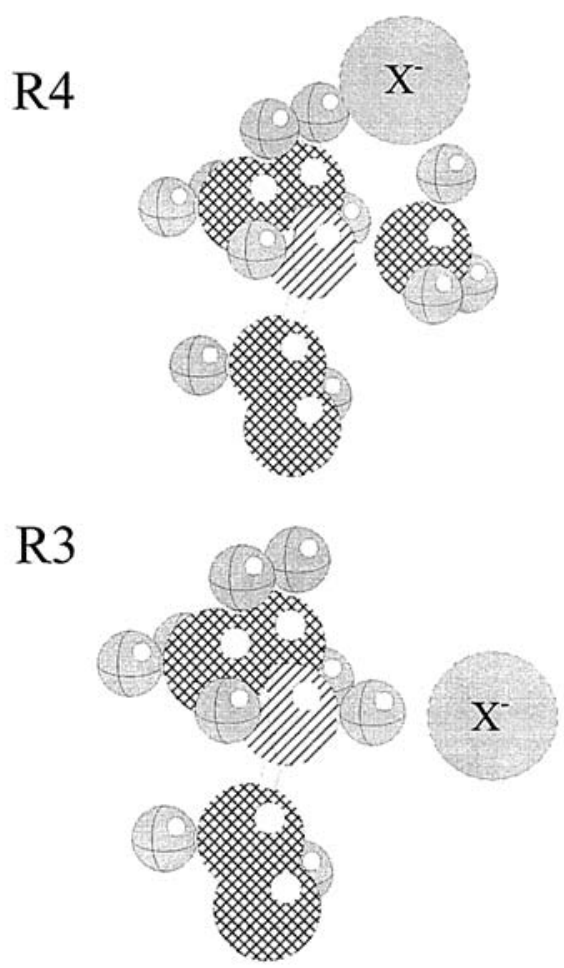

Fig. 2 Possible structures for a halide anions $\left(\mathrm{X}^{-}\right)$in the dried R3 and R4 resins. Reproduced with permission from Ref. 33.

directly interact with the ammonium hydrogen, the ammonium nitrogen should exist in the first coordination shell. The distances between the halide anions and the closest scattering atom are 3.02 and $3.27 \AA$ for $\mathrm{Cl}^{-}$and $\mathrm{Br}^{-}$, respectively, which are shorter than that between the anions and methyl carbon atoms in the R4 resins. The lower figure in Fig. 2 shows a possible local structure of the halide anion in the dried $\mathrm{R} 3$ resin. If the halide anion is directly bound by the ammonium hydrogen, three carbon atoms (two methyl carbon and one methylene carbon atoms) comprise the second coordination shell.

Similarly, the local structures of $\mathrm{K}^{+}, \mathrm{Rb}^{+}$, and $\mathrm{Sr}^{2+}$ in a cationexchange resin were analyzed. ${ }^{34}$ The results are also summarized in Table 1. If a cation is entrapped at the center the

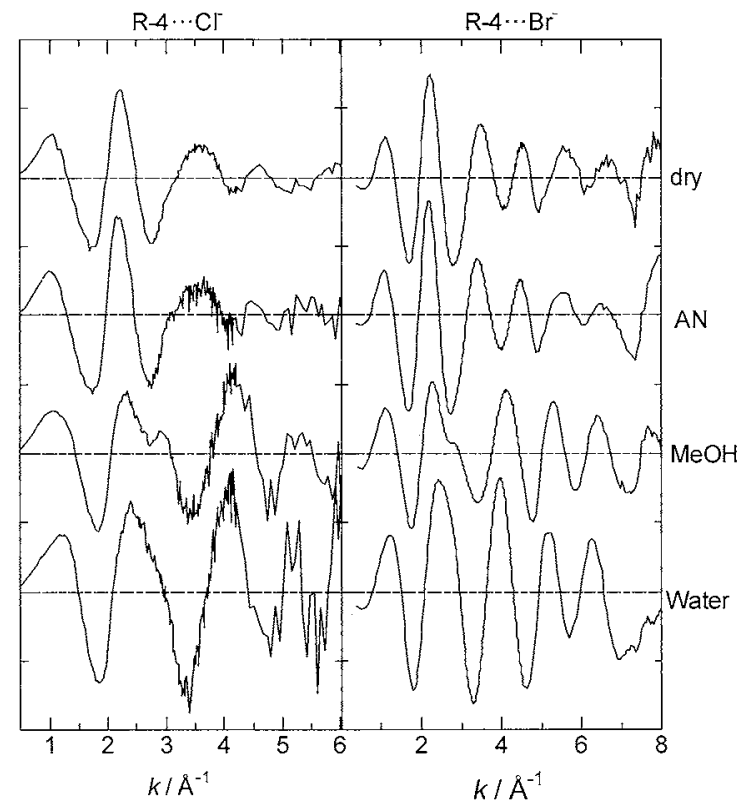

Fig. 3 XAFS $\chi$ spectra of the $\mathrm{R} 3$ and $\mathrm{R} 4$ resins at the $\mathrm{Cl}$ and $\mathrm{Br} K$ edges in various solvents.

tripod of three oxygen atoms in a sulfonate group, a sulfur atom is located slightly farther apart from the cation than the oxygen atoms. Our calculation has indicated that the contribution from a sulfur atom to a spectrum is equivalent to that of an oxygen atom in a sulfonate group. If a cation is entrapped by an $-\mathrm{SO}_{3}$ group, $N$ should be four from this consideration. The coordination numbers determined by spectral analyses were $N=$ 3.1 for $\mathrm{K}^{+}, N=3.7$ for $\mathrm{Rb}^{+}$, and $N=3.7$ for $\mathrm{Sr}^{2+}$. Although the coordination numbers are slightly smaller than 4 , most of the countercations form 1:1 ion pairs with the $-\mathrm{SO}_{3}{ }^{-}$groups. $\mathrm{Sr}^{2+}$ is divalent, and, thus, an intuitive stoichiometric consideration may suggest that two sulfonate groups are detected near the ion. However, interestingly, the XAFS spectra indicate that only one sulfonate group is present in the close vicinity of $\mathrm{Sr}^{2+}$. This is a good example that an inference based on a consideration relying only on electrostatics can lead to misunderstanding. The sulfonate groups should be located at appropriate positions for two sulfonate groups to bind $\mathrm{Sr}^{2+}$ simultaneously. The movement of the sulfonate groups to the appropriate positions to bind multivalent cations may be severely restricted in the macroreticular resins. However, the entire charges in a resin particle should basically be balanced by the stoichiometric ratio of the $-\mathrm{SO}_{3}{ }^{-}$groups and $\mathrm{Sr}^{2+}$.

\subsection{Hydration structures of counterions in ion-exchange resins}

The XAFS spectra for $\mathrm{Cl}^{-}$and $\mathrm{Br}^{-}$in the $\mathrm{R} 4$ resins soaked in various solvents are depicted in Fig. 3. ${ }^{35}$ The spectra obtained for the resin soaked in aprotic solvents such as DMF, DMSO, acetone, and acetonitrile are almost identical to those for dried resins, suggesting that direct ion-pairs are formed even when the resins are swollen in these solvents. In contrast, the spectral features are entirely different for the resins soaked in methanol or water. The hydration structures of counterions are particularly important for the discussion of separation selectivity of ions because ion-exchange separation is usually carried out in aqueous solutions. However, the usual XAFS analysis routines did not resolve the structural features involved in the spectra illustrated in Fig. 3. 

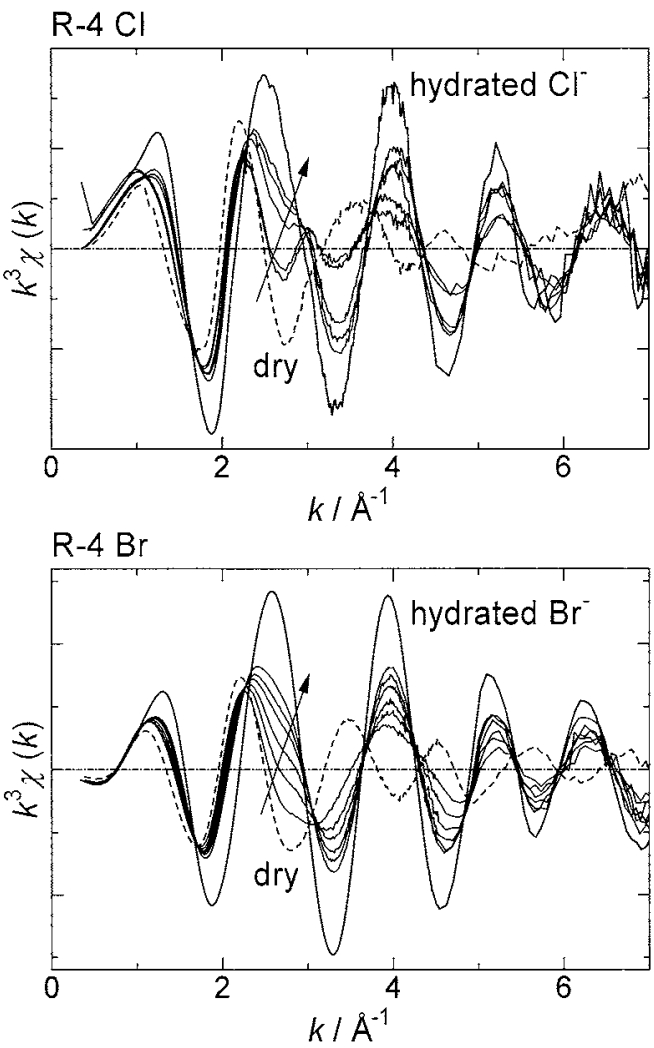

Fig. 4 XAFS $\chi$ spectra for $\mathrm{Cl}^{-}$and $\mathrm{Br}^{-}$dissolved in water and those in the resin equilibrated at various water vapor pressures. Arrows show increasing water content in the resin. Relative water vapor pressures varied: $0.113,0.33,0.582,0.842,0.9$, and 1 . Reproduced with permission from Ref. 36.

Several R4 resin samples having different extents of hydration were prepared by equilibrating the resins under different partial water vapor pressures at $25^{\circ} \mathrm{C} .^{36}$ Figure 4 shows the XAFS spectra of thus prepared resins at the $\mathrm{Cl}$ and $\mathrm{Br}-K$ edges, together with those for dried resins and hydrated $\mathrm{Cl}^{-}$and $\mathrm{Br}-$ for comparison. The hydrated resins have intermediate features between those of dried resins and those of hydrated halide anions, and gradual shifts occur with a change in a water content. The following linear combination was applied to analyze these complex spectra:

$$
\chi=\beta_{1} \chi_{\mathrm{dry}}+\beta_{2} \chi_{\mathrm{hyd}}
$$

where $\chi, \chi_{\text {dry }}$, and $\chi_{\text {hyd }}$ are the $\chi$ spectra obtained for a given resin sample, for the dried resin, and for the hydrated ions, and $\beta_{1}$ and $\beta_{2}$ are coefficients representing the contributions from $\chi_{\text {dry }}$ and $\chi_{\text {hyd }}$, respectively. This equation assumes that the structure of the ion-pair between a counterion and the ammonium ion-exchange group is unchanged after the resin is soaked in water. Table 2 summarized the results of the least squares curve-fitting of Eq. (1). These parameters well reproduce the corresponding experimental spectra. In bulk water, it is known that the hydration number is six for both $\mathrm{Cl}^{-}$ and $\mathrm{Br}^{-}{ }^{37}$ Therefore, $6 \beta_{2}$ represents the average hydration number of the halide anion in the anion-exchange resin. Figure 5 shows the relations between the hydration number of a halide ion $(N)$ and the moles of water adsorbed by an ion-exchange site composed of an ammonium group and a counterion (n). Interestingly, the hydration numbers in the resin first increase with $n$ in a linear fashion but become almost constant after $n$
Table 2 Curve-fitting parameters determined based on Eq. (1) ${ }^{36}$

\begin{tabular}{ccccccccc}
\hline & \multicolumn{7}{c}{$P_{\mathrm{w}} / P_{0}{ }^{\mathrm{a}}$} \\
\cline { 2 - 9 } & & 0.113 & 0.33 & 0.582 & 0.842 & 0.9 & 1 & In water $^{\mathrm{b}}$ \\
\hline $\mathrm{R} 4 \mathrm{Cl}^{\mathrm{c}}$ & $\beta_{1}$ & 0.61 & 0.55 & 0.37 & 0.23 & - & 0.18 & 0.07 \\
& & $(0.15)$ & $(0.15)$ & $(0.15)$ & $(0.15)$ & & $(0.15)$ & $(0.15)$ \\
& $\beta_{2}$ & 0.22 & 0.27 & 0.51 & 0.58 & - & 0.65 & 0.70 \\
& & $(0.07)$ & $(0.07)$ & $(0.07)$ & $(0.07)$ & & $(0.07)$ & $(0.07)$ \\
& $6 \beta_{2}$ & 1.33 & 1.64 & 3.06 & 3.46 & - & 3.90 & 4.20 \\
$\mathrm{R}_{\mathrm{BBr}}{ }^{\mathrm{c}}$ & $\beta_{1}$ & 0.73 & 0.58 & 0.47 & 0.36 & 0.31 & 0.30 & 0.25 \\
& & $(0.22)$ & $(0.22)$ & $(0.22)$ & $(0.22)$ & $(0.22)$ & $(0.22)$ & $(0.22)$ \\
& $\beta_{2}$ & 0.25 & 0.34 & 0.41 & 0.49 & 0.54 & 0.56 & 0.59 \\
& & $(0.08)$ & $(0.08)$ & $(0.08)$ & $(0.08)$ & $(0.08)$ & $(0.08)$ & $(0.08)$ \\
& $6 \beta_{2}$ & 1.49 & 2.04 & 2.47 & 2.92 & 3.22 & 3.37 & 3.55 \\
\hline
\end{tabular}

a. Relative water vapor pressure.

b. Resin soaked in water.

c. Fitting range, $k=1.5-5.5 \AA^{-1}$ for $\mathrm{R} 4 \mathrm{Cl}$ and $k=1.5-6.5 \AA^{-1}$ for $\mathrm{R} 4 \mathrm{Br}$.

Standard deviations in parentheses.

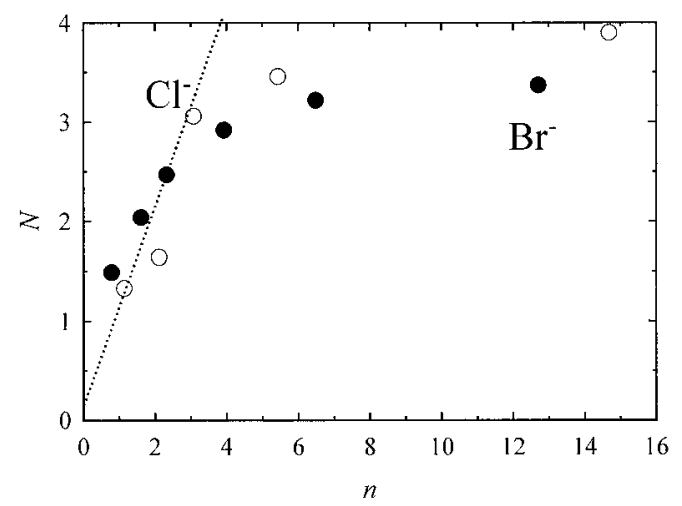

Fig. 5 Relations between $N$ (the hydration number of anions in the resin) and $n$ (the number of water molecules adsorbed by an ionexchange group). Dotted line with a unit slope for eye-guide. Reproduced with permission from Ref. 36.

exceeds $c a$. 3. The hydration numbers of the halide anions in the completely hydrated resins are 3.9 for $\mathrm{Cl}^{-}$and 3.4 for $\mathrm{Br}^{-}$, reflecting different hydration natures of these anions. Detailed analyses of water adsorption isotherms on the resins and these results of XAFS indicated that some of counteranions form direct ion-pairs with the ammonium ion-exchange groups and are simultaneously hydrated by $1-3$ water molecules. In addition, $37 \% \mathrm{Cl}^{-}$and $30 \% \mathrm{Br}^{-}$are completely hydrated even in the resin, and direct ion-pairs are partly dissociated. Yamanaka et al. have indicated that $\mathrm{Cl}^{-}$is partly dehydrated and forms direct ion-pairs with anion-exchange groups by neutron diffraction, albeit the hydration number is different from that determined by XAFS. ${ }^{20}$ Thus, the halide anions are partly dehydrated in anion-exchange resins.

Figure 6 shows the XAFS spectra for $\mathrm{K}^{+}, \mathrm{Rb}^{+}$, and $\mathrm{Sr}^{2+}$ in sulfonate type cation-exchange resins. ${ }^{34}$ The results for dried and hydrated resins are compared with the spectra of the corresponding hydrated ions. The spectrum for hydrated $\mathrm{Rb}^{+}$ perfectly agrees with that for $\mathrm{Rb}^{+}$in the hydrated resin. Similarly, the spectrum for hydrated $\mathrm{Sr}^{2+}$ also completely overlaps with that for $\mathrm{Sr}^{2+}$ in the hydrated resin. These results strongly suggest that these cations are completely hydrated even in the interior of the cation-exchange resins. This is also 


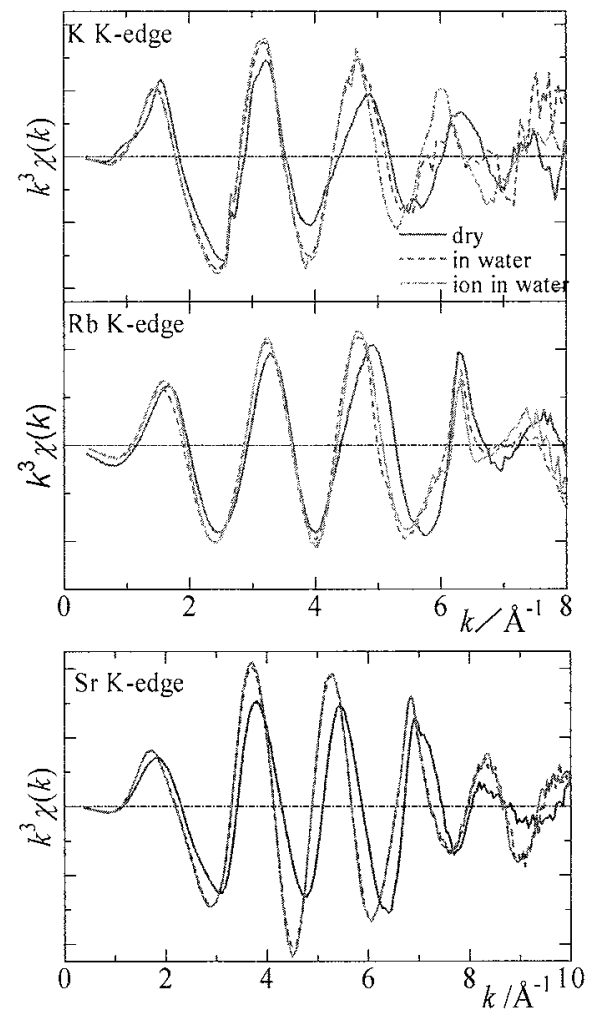

Fig. 6 Comparison of XAFS $\chi$ spectra obtained for $\mathrm{K}^{+}, \mathrm{Rb}^{+}$, and $\mathrm{Sr}^{2+}$ in water and those for the ions in dried and hydrated resins. Reproduced with permission from Ref. 34.

supported by the XANES spectra, in which no clear difference is seen between the hydrated ions and those in hydrated resins. In contrast, the spectrum for $\mathrm{K}^{+}$in the hydrated cation-exchange resin has intermediate spectral features between hydrated $\mathrm{K}^{+}$and $\mathrm{K}^{+}$in the dried resin. The $\chi$ spectrum obtained for $\mathrm{K}^{+}$in the hydrated resin overlaps with that for the hydrated ion in the range of $k<4.5 \AA^{-1}$, but has features for $\mathrm{K}^{+}$in the dried resin for $k>4.5 \AA^{-1}$, suggesting that two major scattering paths are involved. Similarly, the XANES spectrum for hydrated resin showed an intermediate feature between those of dried resin and those of hydrated $\mathrm{K}^{+}$. Thus, Eq. (1) was applied to the $\chi$ spectrum at the $\mathrm{K}-K$ edge for the hydrated cation-exchange resin. Again, the curve-fitting well explains the spectrum with $\beta_{1}=0.40$ and $\beta_{2}=0.63$, implying that some of the potassium ions are directly bound to the sulfonate groups in the resin, while a larger part is completely hydrated. Ordinary considerations will lead to the idea that poorly hydrated ions should be more easily dehydrated than well hydrated counterparts under the strong influence of an electrostatic field or water-shortage conditions as found for the halide anions. However, less hydrated $\mathrm{Rb}^{+}$keeps its hydration shell, whereas more hydrated $\mathrm{K}^{+}$is partly dehydrated. We do not have reasonable explanations for these results at the present stage.

\section{Hydration of Ions in Self-assembled Systems}

3.1 Binding of ions to micelles and their hydration structures in micelles

Micelles are self-assembled molecular aggregates formed by amphiphilic molecules and have been utilized in analytical chemistry as media for separation and recognition of various molecules. To design efficient analytical systems, one must understand the physicochemical nature of micelles. Micellar chromatography and micellar electrokinetic chromatography (MEKC) are typical examples, where micelles play an essential role in the determination of separation performance and selectivity. ${ }^{38-41}$ Sodium dodecylsulfate (SDS) is, for example, most widely applied in MEKC, because the negative zeta potential of the micelle allows us to keep a sufficiently large separation window. In addition, the adsorption of SDS molecules on the capillary wall effectively shortens the total analysis time. These electrokinetic properties of the interfaces involved in the system warrant the wide applicability of this method. The electrokinetic properties of micelles strongly depend on the nature of the counterions. Their binding to the micelle and hydration therein should be understood for successful analytical applications.

In micellar chemistry, the dissociation degree of a micelle $(\alpha)$ has been often used for discussing micellar properties.

$$
[\mathrm{X}]_{\mathrm{free}}=C_{\mathrm{CMC}}+\alpha\left(C-C_{\mathrm{CMC}}\right)
$$

where $[\mathrm{X}]_{\text {free }}, C$ and $C_{\mathrm{CMC}}$ are the concentration of free counterions, the total concentration of a surfactant, and its critical micellar concentration (CMC). It is known that $\alpha$ is constant and independent of $C$, but strongly depends on the natures of the surfactant as well as on those of the counterion. Potentiometry with an electrode selective to a counterion under investigation is usually employed for the determination of $\alpha$. The $\alpha$ values have, for example, been reported to be $0.18-0.23$ and $0.47-0.58$ for dodecyltrimethylammonium bromide (DTAB) and DTA chloride micelles, respectively, and those for hexadecyltrimethylammonium bromide (HTAB) and HTA chloride micelles were reported to be $0.11-0.17$ and 0.24 $0.38 .42-45$ In general, the $\alpha$ value becomes smaller with increasing hydrocarbon chain length for the same counterion. Similarly, the micelle with poorly hydrated counterions shows a smaller $\alpha$ value than the micelle formed by the same amphiphiles with well hydrated counterions.

The dissociation degree is related to the zeta potential of micelles. ${ }^{46-49}$ The zeta potential of a micelle is one of the most useful physicochemical properties of a micelle. Although there is a debate on whether the shear plane corresponds to the real surface or not, the rigorous definition of the micellar surface is of no physical significance because micelles are dynamic systems that are subject to continuous thermal perturbation. The zeta potential, in general, accounts well for the dissociation degree of counterions from the micelle, and thus provides the information of ionic binding to the micelle. Another advantage of the zeta potential is that the discussion based on the electrostatic theory is possible, which enables quantitative evaluation of the ionic interaction occurring on a micelle. A simple mathematical calculation in electrostatics allows us to convert the zeta potential to the charge excess of a spherical micelle. The zeta potential of an ionic micelle generally corresponds to an excess of $c a .10$ charges per micelle. Assuming that the aggregation number of a micelle is 50, this charge excess corresponds to $\alpha=c a$. 0.2 , which well agrees with the potentiometrically determined dissociation degrees discussed above. The zeta potential is thus a reasonable measure for probing the binding of counterions to ionic micelles.

In this review, we will confine our discussions into the micellar interaction with simple inorganic ions to exclude the specific interactions reported e.g. for the interaction of cationic surfactants with aromatic anions. ${ }^{50}$ Ionic surfactants form insoluble salts with poorly hydrated counterions, e.g. $\mathrm{ClO}_{4}{ }^{-}$and 


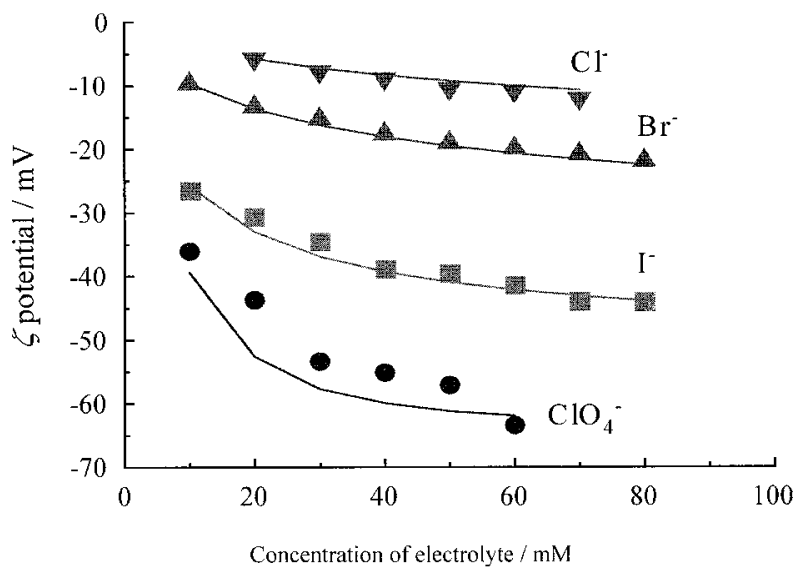

Fig. 7 Comparison of electrophoretically determined zeta potential and calculated surface potential of DDAPS micelles in various electrolytes. The transfer free energy from bulk to the dipolar layer of the micelle: $\Delta \mu_{\mathrm{c}}=0 \mathrm{~kJ} \mathrm{~mol}^{-1}$ for a cation, $\Delta \mu_{\mathrm{a}}=-9.3 \mathrm{~kJ} \mathrm{~mol}^{-1}$ for $\mathrm{ClO}_{4}^{-},-5.0 \mathrm{~kJ} \mathrm{~mol}^{-1}$ for $\mathrm{I}^{-},-0.4 \mathrm{~kJ} \mathrm{~mol}^{-1}$ for $\mathrm{Br}^{-}$and $+2.5 \mathrm{~kJ} \mathrm{~mol}^{-1}$ for $\mathrm{Cl}^{-}$.

$\mathrm{I}^{-}$for cationic surfactants and tetraalkylammonium ion for anionic surfactants. Therefore, the binding abilities of ionic micelles cannot be quantitatively studied over the wide range of the hydration energies of counterions. Nonionic or zwitterionic micelles are free from such restrictions and are suitable for studies of the ionic interaction on micelles without the interference from insoluble salt formation. Dodecyldimethylammoniopropanesulfonate (DDAPS) is the most widely utilized zwitterionic surfactant, having an inner positive group and an outer negative group in a molecule. Since DDAPS is an electrically neutral molecule, a DDAPS micelle also has no nominal electrical charge in pure water. However, in an electrolyte, a relatively large charge is induced by the imbalance between the partition of cations and that of anions into the micelle. ${ }^{46,51}$ Figure 7 summarizes the zeta potential of DDAPS micelles measured in various electrolytes. Interestingly, negative potential is induced in any electrolyte, and the nature of a cation affects the zeta potential only slightly. Thus, the ionic partition into the DDAPS micelles is basically determined by the nature of the anions, and the induced zeta potential decreases in the order of $\mathrm{ClO}_{4}^{-}>\mathrm{I}^{-}>\mathrm{Br}^{-}>\mathrm{Cl}^{-}$, which agrees with the Hofmeister series. The solid curves in Fig. 7 show the results of the calculations based on an electrostatic theory, which involves an ion-pair formation constant of an ion with the DDAPS molecules in the micelle as well as a change in the hydration energy of an ion when it is transferred into the palisade layer of the DDAPS micelle. Interestingly, the zeta potential can be similarly interpreted by assuming either ion-pair formation or hydration change for $\mathrm{ClO}_{4}{ }^{-}$and $\mathrm{I}^{-}$, whereas only the latter explains those for $\mathrm{Br}^{-}$and $\mathrm{Cl}^{-}$. Thus, the zeta potential measurements imply that some anions do not form direct ionpairs with DDAPS molecules in the micelle.

It is thus obvious that the structural and thermodynamic measurements of a single ionic species rather than relative measurements is necessary for further understanding of the capture of ions by micelles. The local structures of bromides on the DTAB and HTAB micelles have been elucidated by XAFS. ${ }^{52}$ Figure 8 shows the spectra obtained for the DTAB micelles with varying the surfactant concentration. The phase shift for $\mathrm{Br}^{-}$on the micelles is similar to that of $\mathrm{Br}^{-}$in bulk water, implying that the dominant scattering path is oxygen atoms in water molecules directly coordinating $\mathrm{Br}^{-}$on the micelles. In contrast, the

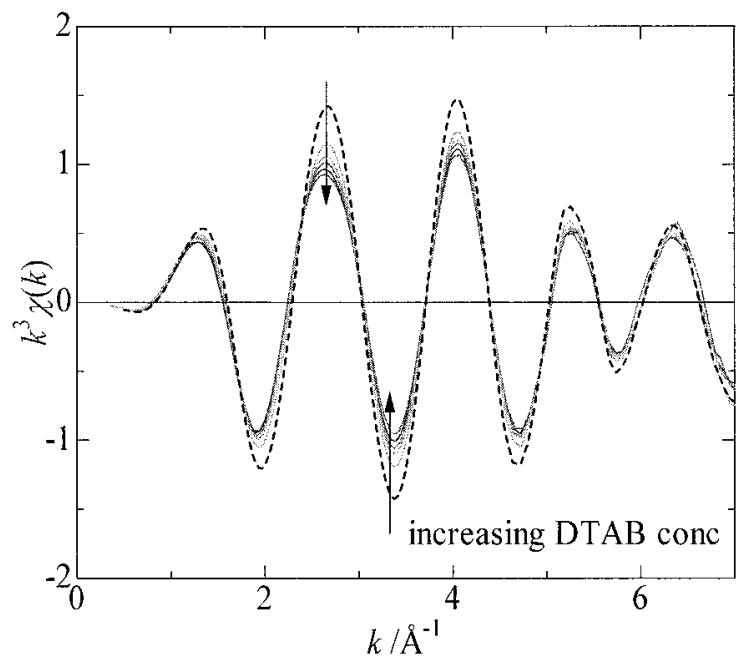

Fig. 8 XAFS $\chi$ spectra for $\mathrm{Br}^{-}$on the DTAB micelle with increasing concentration of the surfactant. Reproduced with permission from Ref. 52 .

oscillation intensity decreases with increasing micellar concentration, indicating that dehydration occurs for bromides bound on the micelles. Interestingly, the extent of the dehydration of the bromides is the same for the DTAB and the HTAB micelles. After the removal of the contribution from free bromides ( $\alpha$ and $C_{\mathrm{CMC}}$ in Eq. (2)), Eq. (1) was applied to the $\chi$ spectra to determine the hydration number of bromides on the micelles. For both the DTAB and HTAB micelles, the hydration number of a bromide was $c a$. 4.3. Assuming that a similar situation for the bromides in anion-exchange resins is applicable to these micelles (fewer than three water molecules hydrate $\mathrm{Br}$ bound on an ammonium group), $c a .40 \%$ of the total bromides partitioned into the cationic micelles are completely hydrated whereas $60 \%$ of them are directly bound to the head groups of the micelle.

The partition of ions into a nonionic micelle should be governed by the solvation energy of ions rather than by electrostatic interactions, because no electrostatic interaction is intrinsically involved in this system; the electrostatic interaction must play some role once the electrostatic potential is induced by the imbalance between cation- and anion-partition. It has been confirmed that negative potential is induced on the nonionic micelle of polyoxyethylene (POE) alkyl ether as summarized in Table 3.47 Again, the same trends predicted by the Hofmeister series, $\mathrm{ClO}_{4}^{-}>\mathrm{I}^{-}>\mathrm{Br}^{-}>\mathrm{Cl}^{-}$, are seen. Although we can estimate the number of charge excess in a micelle, negative zeta potential does not reveal whether the charge excess is caused by the preferable partition of anions or by the exclusion cations from the micelle.

As noted above, the negative zeta potential is generated on this type of nonionic micelle in a usual electrolyte. To discuss this aspect in more detail, we determined the Gibbs free energies of transfer of ions with concentrated aqueous polyethyleneglycol solutions as model of the palisade layer of the POE-type nonionic micelles. Although ion transfer voltammetry is one of the possible methods capable of giving quantitative information on the ionic solvation, the compensation for liquid junction potential is always a problem when the transfer free energy of a single ionic species is discussed. The tetraphenylarsonium tetraphenylborate hypothesis, which has been often employed for this purpose, was not applicable to this particular case. The combined utilization of zeta potential measurements and ion- 
Table 3 The electrophoretic mobility $(\mu)$, zeta potential $(\xi)$, and charge excess $(N)$ of the POE $(6)$ dodecyl ether micelles ${ }^{47}$

\begin{tabular}{|c|c|c|c|}
\hline Electrolyte & $\mu / \mathrm{m}^{2} \mathrm{~s}^{-1} \mathrm{~V}^{-1}$ & $\xi / \mathrm{mV}$ & $N$ \\
\hline $\mathrm{NaClO}_{4}$ & $\begin{array}{l}-5.14 \times 10^{-9} \mathrm{a} \\
\left(1.43 \times 10^{-10}\right)^{\mathrm{b}}\end{array}$ & -9.38 & -4.6 \\
\hline $\mathrm{KClO}_{4}$ & $\begin{array}{l}-5.37 \times 10^{-9} \\
\left(1.79 \times 10^{-10}\right)\end{array}$ & -9.81 & -4.8 \\
\hline $\mathrm{Me}_{4} \mathrm{NClO}_{4}$ & $\begin{array}{c}-5.48 \times 10^{-9} \\
\left(8.20 \times 10^{-11}\right)\end{array}$ & -10.01 & -4.9 \\
\hline $\mathrm{Et}_{4} \mathrm{NClO}_{4}$ & $\begin{array}{c}-5.02 \times 10^{-9} \\
\left(1.16 \times 10^{-10}\right)\end{array}$ & -9.17 & -4.5 \\
\hline $\mathrm{KCl}$ & $\begin{array}{l}-1.09 \times 10^{-9} \\
\left(8.94 \times 10^{-11}\right)\end{array}$ & -2.00 & -0.98 \\
\hline $\mathrm{KBr}$ & $\begin{array}{c}-1.13 \times 10^{-9} \\
\left(1.66 \times 10^{-11}\right)\end{array}$ & -2.07 & -1.0 \\
\hline $\mathrm{Me}_{4} \mathrm{NBr}$ & $\begin{array}{l}-1.79 \times 10^{-9} \\
\left(5.33 \times 10^{-12}\right)\end{array}$ & -3.27 & -1.6 \\
\hline $\mathrm{Et}_{4} \mathrm{NBr}$ & $\begin{array}{l}-1.47 \times 10^{-9} \\
\left(6.80 \times 10^{-11}\right)\end{array}$ & -2.69 & -1.3 \\
\hline
\end{tabular}

a. Negative value means mobility toward the anode. b. Standard deviation.

transfer voltammetry has proven useful to determine the Gibbs free energy of transfer of a single ionic species from water to the interior of the POE-type nonionic micelles $\left(\Delta G_{\mathrm{tr}}^{\circ}\right){ }^{47}$ Selected values are listed in Table 4 . The $\Delta G_{\text {tr }}^{\circ}$ values for $\mathrm{ClO}_{4}^{-}$are negative, whereas those for other ions are positive, suggesting that $\mathrm{ClO}_{4}^{-}$is solvated in the palisade layer of the POE-type micelle better than in water. Also, the $\Delta G_{\text {tr }}^{\circ}$ values for $\mathrm{ClO}_{4}^{-}$, tetraethylammonium ion $\left(\mathrm{Et}_{4} \mathrm{~N}^{+}\right)$, and tetramethylammonium ion $\left(\mathrm{Me}_{4} \mathrm{~N}^{+}\right)$are almost constant with changes in tetraethyleneglycol (tetraEG) concentration. These ions are dominantly hydrated rather than solvated by tetraEG, and, thus, they should compete for water molecules for hydration with hydrophilic tetraEG Since the hydration of $\mathrm{ClO}_{4}^{-}, \mathrm{Et}_{4} \mathrm{~N}^{+}$, and $\mathrm{Me}_{4} \mathrm{~N}^{+}$is weaker than that of other ions listed in Table 4, the effect of the presence of tetraEG may not be very serious; even in the presence of a large amount of tetraEG, these ions can weakly interact with water molecules solvating tetraEG. XAFS measurements in a concentrated polyethyleneglycol as a model solution have indicated that the positive values for $\mathrm{Br}^{-}$partly come from the replacement of water molecules in its first coordination shell by the terminal hydroxyl group in the POE chain..$^{53}$ Thus, when the replacement in the first coordination shell occurs, the $\Delta G_{\mathrm{tr}}^{\circ}$ values are also varied as shown in Table 4 . We cannot directly probe the hydration shells of $\mathrm{ClO}_{4}^{-}, \mathrm{Et}_{4} \mathrm{~N}^{+}$, and $\mathrm{Me}_{4} \mathrm{~N}^{+}$by XAFS. Therefore, molecular simulation should be employed to interpret these findings.

\subsection{Hydration of ions in surface monolayers}

Surfactant molecules are spontaneously adsorbed on the surface of a solution to form a Gibbs monolayer when dissolved in water. Similarly, amphiphiles that are insoluble in water form a Langmuir monolayer when spread on the surface of an aqueous solution. In both cases, amphiphilic molecules are confined within the two-dimensional space between a subphase and air. This type of self assembled system has been studied mainly by thermodynamic measurements such as surface tension measurements for a long time. For ionic surface monolayers, counterions are distributed along the one-dimensional electrostatic potential gradient from the surface to the interior of the subphase. Thus, a surface monolayer is an efficient model system to verify electrostatic theories. From this perspective,
Table $4 \Delta_{\mathrm{W}}^{\mathrm{W}^{\prime}} G_{\mathrm{tr}}^{\circ}$ of ions from water (W) to aqueous tetraethyleneglycol (tetraEG) solutions $\left(\mathrm{W}^{\prime}\right)$ as models of the palisade layer of POE-type nonionic micelles ${ }^{47}$

\begin{tabular}{|c|c|c|c|}
\hline \multirow{3}{*}{ Ion } & \multicolumn{3}{|c|}{$\Delta_{\mathrm{W}}^{\mathrm{W}^{\prime}} G_{\mathrm{tr}}^{\circ} / \mathrm{kJ} \mathrm{mol}^{-1}$} \\
\hline & \multicolumn{3}{|c|}{ TetraEG concentration, $\%$} \\
\hline & 30 & 40 & 50 \\
\hline $\mathrm{Br}^{-}$ & -1.1 & 0.4 & 2.5 \\
\hline $\mathrm{ClO}_{4}^{-}$ & -3.4 & -3.3 & -3.3 \\
\hline $\mathrm{Et}_{4} \mathrm{~N}^{+}$ & 3.5 & 3.7 & 3.5 \\
\hline $\mathrm{Me}_{4} \mathrm{~N}^{+}$ & 4.1 & 4.4 & 4.5 \\
\hline $\mathrm{Cs}^{+}$ & 6.4 & 7.7 & 9.5 \\
\hline $\mathrm{K}^{+}$ & 13.8 & 15.7 & 17.6 \\
\hline
\end{tabular}

Standard deviations of $\Delta_{\mathrm{W}}^{\mathrm{W}^{\prime}} G_{\mathrm{tr}}^{\circ}$ values are $0.3-0.4 \mathrm{~kJ} \mathrm{~mol}^{-1}$.

the direct measurements of the ionic distribution in the vicinity of a charged surface are of fundamental interest. Another point of interest is the local structure of counterions electrostatically attracted by a charged surface monolayer, which can be regarded as an ion-exchange membrane with a regularly aligned molecular structure. ${ }^{54}$ If a surface monolayer well mimics an ion-exchanger, we can evaluate the separation capability of ionexchangers without actually synthesizing them. In addition, a Langmuir monolayer has an advantage that the molecular density can easily be varied by compressing it with an appropriate barrier. ${ }^{55}$ Thus, the distribution of ions just below a surface layer and the local structures of ions deserve investigating in detail not only from the physicochemical viewpoint but also from analytical perspectives. ${ }^{56,57}$

Total reflection XAFS has allowed us to study the aspects associated with the distribution and structure of counterions attracted by a surface monolayer. This method detects the ions present just below a surface monolayer; the detection depth is typically $\sim 10 \mathrm{~nm}$. Therefore, dominant signals come from the ions interacting with or attracted by a surface monolayer. The ion-exchange selectivity of $\mathrm{Br}^{-}$over $\mathrm{Cl}^{-}$was studied on a Langmuir monolayer of ammonium ions having two long hydrocarbon chains with total reflection XAFS. When the surface monolayer is compressed, the selectivity of $\mathrm{Br}^{-}$over $\mathrm{Cl}^{-}$ is enhanced. $\mathrm{Cl}^{-}$with a larger hydrated radius must be squeezed out from the vicinity of the surface monolayer when the monolayer is compressed. ${ }^{54}$ This result strongly implies that these ions are hydrated even when interacting with the positively charged surface monolayer. However, effects of surface monolayer densities and X-ray reflections should be corrected for detailed interpretation of the XAFS spectra. Similarly, combined measurements of surface tension with total reflection XAFS have revealed various thermodynamic features of Gibbs monolayers of cationic surfactants. ${ }^{58-61}$ However, due to the lack of spatial resolution in the perpendicular direction, this method has not been applied to the verification of the dependency of ionic concentration on the distance from the charged layer. Thus, total reflection XAFS is an unusual method that can probe the nanospace just below the surface monolayer; it is expected to reveal the structure of an electrical double layer.

Structural studies of ions attracted by a surface monolayer with total reflection XAFS have been very few, possibly because of the limited quality of spectra obtained thereby. In earlier work, the local structure of $\mathrm{Br}^{-}$attracted by a cation surfactant Gibbs monolayer was studied. A spectrum having similar features to those shown in Figs. 3 and 4 was analyzed by a usual XAFS analysis routine, and a double shell structure, in which a 
Table 5 Hydration numbers of $\mathrm{Br}^{-}$under various circumstances

\begin{tabular}{llc}
\hline Structure of charged group & Type of medium & $\begin{array}{c}\text { Hydration } \\
\text { number }\end{array}$ \\
\hline$-\mathrm{N}^{+}\left(\mathrm{CH}_{3}\right)_{3}$ & Anion-exchange resin & $3.4^{\mathrm{a}}$ \\
$-\mathrm{N}^{+}\left(\mathrm{CH}_{3}\right)_{3}$ & Micelle & $4.3^{\mathrm{b}}$ \\
$-\mathrm{N}^{+}\left(\mathrm{CH}_{3}\right)_{2}-\left(\mathrm{CH}_{2}\right)_{2}-\mathrm{N}^{+}\left(\mathrm{CH}_{3}\right)_{2}-$ & Micelle & $3.5^{\mathrm{c}}$ \\
$\left(\mathrm{CH}_{2}\right)_{3}-\mathrm{SO}_{3}^{-}$ & & \\
$-\mathrm{N}^{+}\left(\mathrm{CH}_{3}\right)_{2}-\left(\mathrm{CH}_{2}\right)_{4}-\mathrm{SO}_{3}{ }^{-}$ & Surface monolayer & $3.5^{\mathrm{d}}$ \\
$\mathrm{N}^{+}\left(\mathrm{C}_{16} \mathrm{H}_{3}\right)_{2}\left(\mathrm{CH}_{3}\right)_{2}$ & Surface monolayer & $6^{\mathrm{e}}$ \\
\hline
\end{tabular}

a. Taken from Ref. 36 .

b. Taken from Ref. 52 .

c. Taken from Ref. 64.

d. Taken from Ref. 63.

e. Unpublished work.

bromide ion was sandwiched by two surfactant molecules, was proposed. $^{62}$ Although this analysis strongly relied on the assumption that long-distance structures were reflected in the spectrum, it is generally difficult to determine such a long distance based on the XAFS spectra obtained for species in a solution. In addition, since it has been elucidated that several $\mathrm{Br}^{-}$species are coexistent in anion-exchange resins and micelles as discussed above, similar structures may be involved for $\mathrm{Br}$ attracted to the Gibbs monolayer of a cationic surfactant. The reevaluation of this spectrum must be necessary.

The local structures of $\mathrm{Br}^{-}$attracted by zwitterionic DDAPS Gibbs monolayer have been analyzed on the basis of the assumption that more than one species are coexistent. ${ }^{63}$ The spectra were successfully interpreted by assuming that $\mathrm{ca}$. $60 \%$ of bromides form direct ion-associates and $c a .40 \%$ of them are hydrated. Thus, a major species of $\mathrm{Br}^{-}$attracted by the monolayer is the ion-associate with the ammonium ion rather than the hydrated ion. In contrast, it has been shown that $\mathrm{Zn}^{2+}$ similarly attracted by the DDAPS monolayer is completely hydrated. As discussed for the hydration structures of counterions in ion-exchange resins, different situations for cations and anions are confirmed for surface monolayer as well. Thus, the total reflection XAFS gives a useful guideline when novel materials are developed for separating and sensing ions.

\section{Ion Recognition Selectivity}

The XAFS data for $\mathrm{Br}^{-}$in various nanospaces, in which ion recognition occurs, are available for comprehensive discussions. The hydration numbers of $\mathrm{Br}^{-}$under various circumstances are summarized in Table 5. We have also added some unpublished data therein. A comparison with the hydration number of $\mathrm{Br}^{-}$in bulk water $(N=6)$ clearly indicates that $2-3$ water molecules are stripped off from the first hydration shell of $\mathrm{Br}^{-}$in most cases. $\mathrm{Br}^{-}$attracted by surface monolayers and micelles must be exposed into the solution in comparison with the inside of ionexchange resins. Therefore, regardless of the structures of ammonium groups, the hydration numbers for the former are larger than that for the latter. Also, effects of the structures of anion-exchange groups can be seen; zwitterionic surfactant molecules appear to facilitate the dehydration of $\mathrm{Br}^{-}$in comparison with the trimethylammonium group. ${ }^{63-65}$ This implies that $\mathrm{Br}^{-}$is more effectively shielded from a bulk solution by the propylene sulfonate groups. In particular, an NMR study has elucidated that $\mathrm{Br}^{-}$is delocalized on the inside ammonium group in the $N$-dodecyl- $N, N^{\prime}$-tetramethylethylendiammonium$N^{\prime}$-propane sulfonate micelle. ${ }^{64}$ Thus, $\mathrm{Br}^{-}$is taken up into the interior of the monolayer or aggregates of the zwitterionic surfactants and is dehydrated to a greater extent possibly because of water-shortage circumstances.

Another important aspects is a difference in solvation structures between anions and cations when they are electrostatically attracted into nanospaces; anions are tend to be dehydrated, while cations generally keep their first hydration shells. Although the molecular mechanisms for these findings have not been fully understood, the binding of a single water molecule to a cation must be stronger than that to an anion as far as alkali cations and halide anions are concerned. This is supported by their hydration numbers; the hydration number of a halide anion is in general larger than that of an alkali cation. These general differences in the structures should be taken into account when a molecular system is designed for recognition and separation of ions.

If the first hydration shells are maintained, what is the origin in the recognition selectivity of identically charged ions? Recently, Ohki et al. estimated the contribution from the second or farther hydration shell to the entire hydration energies of alkali cations and bromide ion by ion-transfer voltammetry and XAFS. ${ }^{6}$ According to this study, the contribution from the second or farther shells is larger than $10-15 \mathrm{~kJ} \mathrm{~mol}^{-1}$, corresponding to the energy of a single hydration bond formation. In addition, the energy of the perturbation becomes larger for smaller alkali cations. When ion recognition occurs in nanospaces or near the interface, the second or further hydration shell of an ion should be perturbed more easily than that of the first hydration shell. This perturbation energy is smaller than $10 \%$ of the total hydration energy of an ion but is large enough to explain an energetic difference in ion recognition selectivity; for example, the energy of ion-exchange between $\mathrm{K}^{+}$and $\mathrm{Rb}^{+}$or $\mathrm{Cl}^{-}$and $\mathrm{Br}^{-}$is smaller than $2-3 \mathrm{~kJ} \mathrm{~mol}^{-1}$.

\section{Conclusion}

The hydration of ions in confined spaces, such as the interior of ion-exchange resins and molecular aggregates, has been discussed on the bases of our work on XAFS, electrophoresis, and ion-transfer voltammetry. Due to the limitation of the applicability of XAFS, the discussions have focused mainly on the local structures of $\mathrm{Br}^{-}$under various circumstances. However, the results of two or more ions are necessary for discussing selectivity, and, from this perspective, our work should be extended to the elements located above or below bromine on the periodical table. Another important direction is that the results shown here can be effectively utilized for designing a system with ionic selectivity that established systems do not have. We hope that studies on molecular bases will become recognized as a more efficient way than conventional trials and errors in the near future.

\section{References}

1. P. Selvam, S. K. Bhatia, and C. G. Sonwane, Ind. Eng. Chem. Res., 2001, 40, 3237.

2. M. Oguni, Y. Kanke, and S. Namba, AIP Conf. Proc., 2008, 982, 34

3. S. Takahara, N. Sumiyama, S. Kittaka, T. Yamaguchi, and M.-C. Bellissent-Funel, J. Phys. Chem. B, 2005, 109, 11231.

4. S. Kittaka, S. Takahara, T. Yamaguchi, and M.-C. Bellissent Funel, Langmuir, 2005, 21, 1389. 
5. T. Takamuku, H. Maruyama, S. Kittaka, S. Takahara, and T. Yamaguchi, J. Phys. Chem. B, 2005, 109, 892.

6. T. Tsukahara, A. Hibara, Y. Ikeda, and T. Kitamori, Angew. Chem., Int. Ed., 2007, 46, 1180.

7. H. Yui, Y. Guo, K. Koyama, T. Sawada, G. John, B. Yang, M. Masuda, and T. Shimizu, Langmuir, 2005, 21, 721.

8. E. Fois, A. Gamba, E. Spano, and G. Tabacchi, J. Mol. Struct., 2003, 644, 55.

9. M. Carrillo-Tripp, M. L. San-Román, J. Hernańdez-Cobos, H. Saint-Martin, and I. Ortega-Blake, Biophys. Chem., 2006, 124, 243.

10. B. Schnell, R. Schurhammer, and G. Wipff, J. Phys. Chem. $B$, 2004, 108, 2285.

11. T. Ohkubo, Y. Hattori, H. Kanoh, T. Konishi, T. Fujikawa, and K. Kaneko, J. Phys. Chem. B, 2003, 107, 13616.

12. "Interfacial Nanochemistry: Molecular Science and Engineering at Liquid-Liquid Interfaces", ed. H. Watarai, N. Teramae, and T. Sawada, 2005, Academic/Plenum Publishers, New York, Kluwer, 205.

13. H. Watarai (Guest Editor), Anal. Sci., 2004, 20, No. 11.

14. T. Okada and M. Harada, Bunseki Kagaku, 2005, 54, 27.

15. D. Reichenber, in "Ion Exchange. A Series of Advances", 1966, ed. J. A. Marinsky, Vol. 1, Marcle Dekker, New York, 227.

16. D. Nandan and A. R. Gupta, J. Phys. Chem., 1975, 79, 180.

17. D. Nandan and A. R. Gupta, J. Phys. Chem., 1977, 81, 1977.

18. D. Nandan, B. Venkataramani, and A. R. Gupta, Langmuir, 1993, 9, 1786.

19. R. S. D. Toteja, B. L. Jangida, M. Sundaresan, and B. Venkataramani, Langmuir, 1997, 13, 2980.

20. K. Yamanaka, Y. Kameda, Y. Amo, and T. Usuki, J. Phys. Chem. B, 2007, 111, 11337.

21. Y. Kameda, K. Yamanaka, M. Sasaki, Y. Amo, and T. Usuki, Bull. Chem. Soc. Jpn., 2006, 79, 1032.

22. R. H. Tromp and G. W. Neilson, J. Phys. Chem., 1996, 100, 7380.

23. V. Crupi, F. Longo, D. Majolino, and V. Venuti, J. Chem. Phys., 2005, 123, 154702.

24. Yu. V. Lisichkin, L. A. Sakharova, and A. A. Tumanov, Crystallogr. Rep., 2007, 52, 618.

25. S. Crouzy, J. Phys. IV, 2005, 130, 179.

26. J. W. Kang, K. R. Byun, J. Y. Lee, S. C. Kong, Y. W. Choi, and H. J. Hwang, Phys. E, 2004, 24, 349.

27. B. Rotenberg, V. Marry, R. Vuilleumier, N. Malikova, C. Simon, and P. Turq, Geochim. Cosmochim. Acta, 2007, 71, 5089.

28. H.-G. Haubold, Th. Vad, H. Jungbluth, and P. Hiller, Electrochim. Acta, 2001, 46, 1559.

29. T. D. Gierke and W. Y. Hsu, in "Perfluorinated Ionomer Membranes", 1982, ed. A. Eisenberg and H. L. Yeager, American Chemical Society, ACS Symposium Series 180, 283

30. A. Vishnyakov and A. V. Neimark, J. Phys. Chem. B, 2000, 104, 4471.

31. D. L. Duval and J. S. Fritz, J. Chromaogr., 1984, 295, 89.

32. "Ion Koukan", 1991, ed. M. Senou, M. Abe, and T. Suzuki, Kodansha, Tokyo.

33. M. Harada and T. Okada, J. Chromatogr., A, 2005, 1085, 3.

34. M. Harada and T. Okada, Chem. Commun., 2008, 5182.
35. M. Harada, T. Okada, and I. Watanabe, J. Phys. Chem. B, 2002, 106, 34.

36. T. Okada and M. Harada, Anal. Chem., 2004, 76, 4564.

37. H. Ohtaki and T. Radnai, Chem. Rev., 1993, 93, 1157.

38. K. Isoo and S. Terabe, Anal. Sci., 2005, 21, 43.

39. K. Sueyoshi, F. Kitagawa, and K. Otsuka, Anal. Chem., 2008, 80, 1255.

40. Y. Esaka, M. Sawamura, H. Murakami, and B. Uno, Anal. Chem., 2006, 78, 8142.

41. K. Iso and T. Okada, J. Chromatogr., A, 2001, 920, 317.

42. T. Asakawa, H. Kitano, A. Ohta, and S. Miyagishi, J. Colloid Interface Sci., 2001, 242, 284.

43. S. E. Moore, M. Mohareb, S. A. Moore, and R. M. Palepu, J. Colloid Interface Sci., 2006, 304, 491.

44. R. Zana, J. Colloid Interface Sci., 1980, 78, 330.

45. L. Gaillon, J. Lelièvre, and R. Gaboriaud, J. Colloid Interface Sci., 1999, 213, 287.

46. K. Iso and T. Okada, Langmuir, 2000, 16, 9199.

47. T. Ohki, M. Harada, and T. Okada, J. Phys. Chem. B, 2006, 110, 15486.

48. R. Sabaté, M. Gallardo, and J. Estelrich, Electrophoresis, 2000, $21,481$.

49. M. Johnsson and J. B. F. N. Engbets, J. Phys. Org. Chem., 2004, 17, 934.

50. M. A. Cassidy and G. G. Warr, J. Phys. Chem., 1996, 100, 3237.

51. T. Masudo and T. Okada, Phys. Chem. Chem. Phys., 1999, 1,3577 .

52. M. Harada, H. Satou, and T. Okada, J. Phys. Chem. B, 2007, 111, 12136.

53. T. Ohki, M. Harada, and T. Okada, J. Phys. Chem. B, 2007, 111,7245 .

54. M. Harada and T. Okada, Langmuir, 2004, 20, 30.

55. M. Harada, T. Okada, and I. Watanabe, Anal. Sci., 2002, 18, 1167.

56. M. Harada and T. Okada, Hyomen, 2005, 43, 487.

57. M. Harada, T. Okada, H. Tanida, and I. Watanabe, Bunseki Kagaku, 2003, 52, 405.

58. K. Kashimoto, K. Shibata, T. Matsuda, M. Hoshide, Y. Jimura, I. Watanabe, H. Tanida, H. Matsubara, T. Takiue, and H. Aratono, Langmuir, 2008, 24, 6693.

59. K. Kashimoto, Y. Takata, T. Matsuda, N. Ikeda, H. Matsubara, T. Takiue, M. Aratono, H. Tanida, and I. Watanabe, Langmuir, 2006, 22, 8403.

60. M. Aratono, K. Kashimoto, T. Matsuda, S. Muroi, Y. Takata, N. Ikeda, T. Takiue, H. Tanida, and I. Watanabe, Langmuir, 2005, 21, 7398.

61. T. Takiue, Y. Kawagoe, S. Muroi, R. Murakami, N. Ikeda, M. Aratono, H. Tanida, H. Sakane, M. Harada, and I. Watanabe, Langmuir, 2003, 19, 10803.

62. I. Watanabe and H. Tanida, Anal. Sci., 1995, 14, 525.

63. M. Harada, T. Okada, and I. Watanabe, J. Phys. Chem. B, 2003, 107, 2275.

64. T. Aoki, M. Harada, and T. Okada, Langmuir, 2007, 23, 8820.

65. T. Aoki, M. Harada, and T. Okada, Langmuir, 2007, 23, 12473.

66. T. Ohki, M. Harada, and T. Okada, J. Phys. Chem. B, 2008, $112,11863$. 